\title{
A New lifetime model for multivariate survival data with a surviving fraction
}

\author{
Vicente G. Cancho, Francisco Louzada, Dipak K. Dey \& Gladys D.C. Barriga
}

To cite this article: Vicente G. Cancho, Francisco Louzada, Dipak K. Dey \& Gladys D.C. Barriga (2016) A New lifetime model for multivariate survival data with a surviving fraction, Journal of Statistical Computation and Simulation, 86:2, 279-292, DOI: 10.1080/00949655.2015.1007983

To link to this article: https://doi.org/10.1080/00949655.2015.1007983

册 Published online: 06 Feb 2015.

Submit your article to this journal

Џll Article views: 257

View Crossmark data $־$

Citing articles: 1 View citing articles 


\title{
A New lifetime model for multivariate survival data with a surviving fraction
}

\author{
Vicente G. Cancho ${ }^{\mathrm{a}}$, Francisco Louzada* ${ }^{\mathrm{a}}$, Dipak K. Dey ${ }^{\mathrm{b}}$ and Gladys D.C. Barriga ${ }^{\mathrm{c}}$ \\ ${ }^{a}$ ICMC, Universidade de São Paulo, São Carlos, Brazil; ${ }^{b}$ Department of Statistics, University of \\ Connecticut, Storrs, CT, USA; ${ }^{c}$ FEB, State Universidade de São Paulo, Bauru, Brazil
}

(Received 27 April 2014; accepted 12 January 2015)

\begin{abstract}
In this paper we propose a new lifetime model for multivariate survival data with a surviving fraction. We develop this model assuming that there are $m$ types of unobservable competing risks, where each risk is related to a time of the occurrence of an event of interest. We explore the use of Markov chain Monte Carlo methods to develop a Bayesian analysis for the proposed model. We also perform a simulation study in order to analyse the frequentist coverage probabilities of credible interval derived from posteriors. Our modelling is illustrated through a real data set.
\end{abstract}

Keywords: Bayesian inference; competing risks; MCMC; multivariate survival models; cure rate models; cured fraction

\section{Introduction}

Cure rate models play an important role in survival analysis, covering data where a proportion of subjects may remain susceptible to the event of interest. These models, also referred to as survival models with a surviving fraction or long-term survival models, have become very popular due to significant progress and advancements in treatment therapies leading to enhanced cure rates. The proportion of these 'cured' units is termed the cured fraction. The terminology is based on clinical studies, where the event of interest can be the death of a patient or a tumour recurrence (which can be attributed to metastasis-component tumour cells left active after an initial treatment), which cannot occur due to the event of interest. However, nowadays, it is common to observe applications of cure rate models studies of many other kinds of events, such as criminal recidivism, divorce, child-bearing, unemployment and costumer churn, amongst others.

Two formulations of cure rate models stand out in the literature as being the prevailing approaches. Here, we point out a distinguishing feature between them. Firstly, the standard mixture cure model, [1,2] where the number of competing causes of the event of interest is a binary random variable on $\{0,1\}$, and recently, the promotion time cure model,[3] where the number of competing causes follows a Poisson distribution. However, these assumptions may not be verified once the number of competing causes is an unobserved random variable (latent variable). In order to overcome this restriction, several proposals appeared in the literature considering general

*Corresponding author. Email: louzada@icmc.usp.br 
distributions, such as the geometric, negative binomial, power series, Conway-Maxwell-Poisson distributions, amongst other. The literature on cure rate models is by now vast and expanding rapidly. The books by Maller and Zhou [4] and Ibrahim et al.,[5] as well as the articles by Chen et al.,[6] Tsodikov et al.,[7] Tournoud and Ecochard,[8] Cancho et al.,[9] Perdona and Louzada-Neto,[10] Kim et al. [11] and Cobre et al. [12] to name a few.

Although extensions of cure rate models were developed, limited attention has been paid to the research on multivariate cure rate models. In the frequentist framework, Chatterjee and Shih [13] proposed a marginal approach using bivariate copula models. Price and Manatunga [14] imposed frailty to account for correlation and conducted the maximum likelihood estimation under a parametric model assumption. Both methods were based on the mixture cure model. In the Bayesian paradigm, Chen et al. [15] generalized the work of Yakovlev and Tsodikov [3] to multivariate failure time data by introducing a positive stable frailty and Louzada et al. [16] proposed bivariate long-term distribution based on the Farlie-Gumbel-Morgenstern copula model.

In this paper, a new multivariate cure rate survival model is developed under a scenario of latent competing causes. In the formulation, we consider that there are $m$ types of latent causes of failures, which can produce the correspondent event of interest, where these latent variables are modelled by a multivariate Poisson distribution.[17] In Section 2, we formulate the multivariate cure rate model. We explore the use of Markov chain Monte Carlo (MCMC) methods to develop a Bayesian analysis in Section 3. Simulation study is presented in Section 4. An application to a real data set is given in Section 5. Finally, Section 6 concludes with some general remarks.

\section{The model}

For an individual in the population, let $N_{k}(k=1, \ldots, m)$ be the random variable that denotes the unobservable number of causes of type $k$ of the event of interest for this individual. We assume that $N=\left(N_{1}, N_{2}, \ldots, N_{m}\right)$ follows a multivariate Poisson distribution with probability mass function

$$
P\left[N_{1}=n_{1}, \ldots, N_{m}=n_{m}\right]=\mathrm{e}^{-\left\{\sum_{i=1}^{m} \theta_{i}\right\}} \prod_{i=1}^{m} \frac{\theta_{i}^{n_{i}}}{n_{i} !} \sum_{i=0}^{s} \prod_{j=1}^{m}\left(\begin{array}{c}
n_{j} \\
i
\end{array}\right) i !\left(\frac{\theta_{0}}{\prod_{i=1}^{m} \theta_{i}}\right)^{i}
$$

where $n_{j}=0,1, \ldots, \theta_{j}>0, j=0,1, \ldots, m$ and $s=\min \left\{n_{1}, \ldots, n_{m}\right\}$. The above multivariate distribution allows for positive dependence between the two random variables. Marginally each random variable follows a Poisson distribution with $E\left(N_{j}\right)=\theta_{j}+\theta_{0}$ and, $\operatorname{Cov}\left(N_{i}, N_{j}\right)=\theta_{0}$, $i \neq j=1, \ldots, m$ and hence $\theta_{0}$ is a measure of dependence between the two random variables. If $\theta_{0}=0$, then the variables are independent and the multivariate Poisson distribution reduces to the product of $m$ independent Poisson distributions. For a comprehensive treatment of the multivariate Poisson distribution, the reader can refer to Karlis.[17] It is to be noted that the $N$-vector also indicates a multivariate frailty which was also considered by Caroni et al.[18] Furthermore, this model arrives as boundary case for the compound Poisson model. Several models of these types were studied in [14].

The time for the $j$ th competing cause of type $k$ to produce the event of interest is denoted by $Z_{k j}, k=1, \ldots, m, j=1,2, \ldots$ Given $N_{k}=n_{k}$, the $Z_{k 1} \ldots, Z_{k n_{k}}$ are independent and identically distributed random variables with cumulative distribution function $F_{k}(\cdot)=1-S_{k}(\cdot)$. We also assumed that the latent variables $Z_{1 j}, \ldots, Z_{k j}$ are independent. The observed times to event are defined by the random variables $Y_{k}=\min \left\{Z_{k 0}, Z_{k 1}, \ldots Z_{k N_{k}}\right\}$ with $P\left(Z_{k 0}=\infty\right)=1$, $k=1,2, \ldots, m$. Under this set-up we can demonstrate, that the population survival function for 
$\boldsymbol{Y}=\left(Y_{1}, \ldots, Y_{m}\right)$ is given by

$$
S_{\text {pop }}(\boldsymbol{y})=\exp \left\{-\sum_{i=1}^{m} \theta_{i}\left(1-S_{i}\left(y_{i}\right)\right)-\theta_{0}\left(1-\prod_{i=1}^{m} S_{i}\left(y_{i}\right)\right)\right\} .
$$

The survival function $S_{\text {pop }}(y)$ in Equation (2) is not a proper survival, that is, $\lim _{y_{1}, \ldots, y_{m} \rightarrow \infty} S_{\text {pop }}(\boldsymbol{y})=\exp \left\{-\sum_{i=0}^{m} \theta_{i}\right\}>0$ (the joint cure fraction). Note that when $\theta_{0}=0$ in Equation (2), the joint survival function reduces to the product of $m$ independent survival functions. From Equation (2), the marginal survival functions are

$$
S_{\text {pop }}\left(y_{k}\right)=\exp \left\{-\left(\theta_{k}+\theta_{0}\right) F_{k}\left(y_{k}\right)\right\}, \quad k=1, \ldots, m .
$$

Equation (3) indicates that the marginal survival function has a cure rate structure with probability of cure $p_{0 k}=\mathrm{e}^{-\theta_{k}-\theta_{0}}$ for $Y_{k}, k=1, \ldots, m$. It is important to note in Equation (3) that each marginal survival function has the structure of the promotion time cure model.[3,6] In Equation (3) that each marginal distribution has a proportional hazards structure as long as the covariates, only enter through $\theta_{k}$ and $\theta_{0}$. The marginal hazard function is given by, $\left(\theta_{k}+\theta_{0}\right) f_{k}\left(y_{k}\right)$ which satisfies the conditions for the proportional hazards model.[19] This is a desirable feature of the proposed model that leads to attractive theoretical properties.

Without loss of generality, considering the bivariate distribution of $\left(Y_{1}, Y_{2}\right)$, then joint survival function is given by

$$
S_{\mathrm{pop}}\left(y_{1}, y_{2}\right)=\exp \left\{-\theta_{1}\left(1-S_{1}\left(y_{1}\right)\right)-\theta_{2}\left(1-S_{2}\left(y_{2}\right)\right)-\theta_{0}\left(1-S_{1}\left(y_{1}\right) S_{2}\left(y_{2}\right)\right)\right\}
$$

This distribution will be called as a bivariate cure rate (BCR) model. The parameter $\theta_{0}$ is a measure of association between $\left(Y_{1}, Y_{2}\right)$. As $\theta_{0} \rightarrow 0$, this implies less association between $\left(Y_{1}, Y_{2}\right)$ which can be seen from Equation (4). Following Clayton [20] and Oakes,[21] we can compute a local measure of association, denoted by $\vartheta^{*}\left(Y_{1}, Y_{2}\right)$, as a function of $\theta_{0}$. This measure of association is defined as

$$
\vartheta^{*}\left(Y_{1}, Y_{2}\right)=\frac{S_{\text {pop }}\left(y_{1}, y_{2}\right)\left(\partial^{2} / \partial y_{1} \partial y_{2}\right) S_{\text {pop }}\left(y_{1}, y_{2}\right)}{\left(\partial S_{\text {pop }}\left(y_{1}, y_{2}\right) / \partial y_{1}\right)\left(\partial S_{\text {pop }}\left(y_{1}, y_{2}\right) / \partial y_{2}\right)} .
$$

The measure in Equation (5), has the interpretation as the ratio of the hazard rate of the conditional distribution of $Y_{1}\left(Y_{2}\right)$, given $Y_{2}=y_{2}\left(Y_{1}=y_{1}\right)$, to that of $Y_{1}\left(Y_{2}\right)$ given $Y_{2}>y_{2}\left(Y_{1}>y_{1}\right)$. For more discussion of Equation (5), see Clayton [20] and Oakes.[21] For the BCR model in Equation (4), $\vartheta^{*}\left(y_{1}, y_{2}\right)$ is well defined and is given by

$$
\left.\vartheta^{*}\left(y_{1}, y_{2}\right)=1+\theta_{0}\left\{\left[\theta_{1}+\theta_{0} S_{2}\left(y_{2}\right)\right]\left[\theta_{2}+\theta_{0} S_{1}\left(y_{1}\right)\right)\right]\right\}^{-1} .
$$

We see that $\vartheta^{*}\left(y_{1}, y_{2}\right)$ in Equation (6) increases in $\left(y_{1}, y_{2}\right)$. That is, the association between $\left(y_{1}, y_{2}\right)$ is less when $\left(y_{1}, y_{2}\right)$ are small and the association increases over time.

The following theorem is a generalization of the proposed model in Equation (2) and provides a natural extension of the univariate survival cure rate models.

THEOREM 2.1 Let $N=\left(N_{1}, N_{2}, \ldots, N_{m}\right)$ be a random vector with probability-generating function, $\varphi_{N}\left(w_{1}, \ldots, w_{m}\right)$ and random vector $\boldsymbol{Y}=\left(Y_{1}, \ldots, Y_{m}\right)$ defined in Equation (2). Then, the joint survival function of $\boldsymbol{Y}$ is given by

$$
S_{p o p}(\boldsymbol{y})=\varphi_{N}\left(S_{1}\left(y_{1}\right), \ldots, S_{m}\left(y_{m}\right)\right)
$$


Proof Under the conditions of theorem, we can write the joint survival function of $\boldsymbol{Y}$ as

$$
\begin{aligned}
S_{\mathrm{pop}}(\boldsymbol{y})= & P[\boldsymbol{N}=\boldsymbol{0}]+\sum_{n_{1}, \ldots, n_{m}=1}^{\infty} P\left[Z_{11}>y_{1}, \ldots, Z_{1 n_{1}}>y_{1}, \ldots, Z_{m 1}>y_{m}, \ldots, Z_{m n_{m}}>y_{m}\right] \\
& \times P\left[N_{1}=n_{1}, \ldots, N_{m}=n_{m}\right] \\
= & P\left[N_{1}=0, \ldots, N_{m}=0\right]+\sum_{n_{1}, \ldots, n_{m}=1}^{\infty} P\left[N_{1}=n_{1}, \ldots, N_{m}=n_{m}\right] S_{1}^{n_{1}}\left(y_{1}\right) \cdots S_{m}^{n_{m}}\left(y_{m}\right) \\
= & \sum_{n_{1}, \ldots, n_{m}=1}^{\infty} P\left[N_{1}=n_{1}, \ldots, N_{m}=n_{m}\right] S_{1}^{n_{1}}\left(y_{1}\right) \cdots S_{m}^{n_{m}}\left(y_{m}\right) \\
= & \varphi_{\boldsymbol{N}}\left(S_{1}\left(y_{1}\right), \ldots, S_{m}\left(y_{m}\right)\right) .
\end{aligned}
$$

The last step comes from the definition of the probability-generating function.

Again the survival function $S_{\mathrm{pop}}(\boldsymbol{y})$ in Equation (7) is not a proper survival, that is, $\lim _{y_{1}, \ldots, y_{m} \rightarrow \infty} S_{\text {pop }}(\boldsymbol{y})=\varphi_{N}(0, \ldots 0)=P\left[N_{1}=0, \ldots, N_{m}=0\right]>0$ (joint cure rate). From Equation (7), the marginal survival function is obtained as

$$
S_{\text {pop }}\left(y_{k}\right)=\varphi_{N_{k}}\left(S_{k}\left(y_{k}\right)\right), \quad k=1, \ldots, m .
$$

The marginal survival function (8) is the same as the one proposed by Tsodikov et al. [7] and Rodrigues et al.[22] The marginal cure rate proportion is $S_{k}(\infty)=\varphi_{N_{k}}(0)=P\left[N_{k}=0\right]>0$. In the case that random variables $N_{1}, \ldots, N_{m}$ are independent, the survival function $S_{\text {pop }}(\boldsymbol{y})$ in Equation (7) is given by

$$
S_{\text {pop }}(\boldsymbol{y})=\prod_{k=1}^{m} \varphi_{N_{k}}\left(S_{k}\left(y_{k}\right)\right) .
$$

The proposed model in Equation (2) can be obtained from Theorem 2.1. To be more specific, the random vector $N=\left(N_{1}, \ldots, N_{m}\right)$ with multivariate Poisson given in Equation (1) is defined as $N_{k}=D_{k}+D_{0}, k=1, \ldots, m$, where $D_{i}, i=0,1, \ldots, m$ are independent univariate Poisson distributed random variables with parameters $\theta_{i}$. The probability-generating function, of $N$ is defined as

$$
\begin{aligned}
\varphi_{N}\left(w_{1}, \ldots, w_{m}\right) & =E\left[w_{1}^{N_{1}} w_{2}^{N_{2}} \cdots w_{m}^{N_{m}}\right]=E\left[w_{1}^{D_{1}+D_{0}} \times w_{2}^{D_{2}+D_{0}} \times \cdots \times w_{m}^{D_{m}+D_{0}}\right] \\
& =E\left[w_{1}^{D_{1}} \times w_{2}^{D_{2}} \times \cdots \times w_{m}^{D_{m}}\left(w_{1} \times \cdots \times w_{m}\right)^{D_{0}}\right] \\
& =\varphi_{D_{1}}\left(w_{1}\right) \times \cdots \times \varphi_{D_{m}}\left(w_{m}\right) \times \varphi_{D_{0}}\left(\prod_{i=1}^{m} w_{i}\right) \\
& =\exp \left\{-\sum_{i=1}^{m} \theta_{i}\left(1-w_{i}\right)-\theta_{0}\left(1-\prod_{i=1}^{m} w_{i}\right)\right\} .
\end{aligned}
$$

From Theorem 2.1 and Equation (9), we obtain the model given in Equation (2).

We can also obtain an extension of bivariate version of the univariate standard mixture cure rate model. In what follows, consider a bivariate Bernoulli random vector $\left(N_{1}, N_{2}\right)$, which takes values from $(0,0),(0,1),(1,0)$ and $(1,1)$ in the cartesian product space $\{0,1\}^{2}=\{0,1\} \times\{0,1\}$ with probability $\theta_{i j}=P\left[N_{1}=i, N_{2}=j\right], i, j=0,1$, such that, $\sum_{i=0}^{1} \sum_{j=0}^{1} \theta_{i j}=1$. Then, the corresponding probability-generating function is given by $\varphi_{N}\left(w_{1}, w_{2}\right)=\theta_{00}+\theta_{10} w_{1}+\theta_{01} w_{2}+$ 
$\theta_{11} w_{1} w_{2}$. From Theorem 2.1, we obtain the following results related to the standard mixture cure rate model:

$$
S_{\text {pop }}\left(y_{1}, y_{2}\right)=\theta_{00}+\theta_{10} S_{1}\left(y_{1}\right)+\theta_{01} S_{2}\left(y_{2}\right)+\theta_{11} S_{1}\left(y_{1}\right) S_{2}\left(y_{2}\right) .
$$

Thus, the joint cure fraction is $S_{\text {pop }}(\infty, \infty)=\theta_{00}$. The marginal survival functions are given by $S_{\text {pop }}\left(y_{1}\right)=\theta_{00}+\theta_{01}+\left(\theta_{10}+\theta_{11}\right) S_{1}\left(y_{1}\right)$ and $S_{\text {pop }}\left(y_{2}\right)=\theta_{00}+\theta_{10}+\left(\theta_{01}+\theta_{11}\right) S_{2}\left(y_{2}\right)$.

Now, if we consider a random vector $\left(N_{1}, N_{2}\right)$ which follows a bivariate geometric distribution with probability mass function

$$
P\left[N_{1}=n_{1}, N_{2}=n_{2}\right]=\left(\begin{array}{c}
n_{1}+n_{2} \\
n_{1}
\end{array}\right)\left(1-\theta_{1}-\theta_{2}\right) \theta_{1}^{n_{1}} \theta_{2}^{n_{2}},
$$

where $n_{j}=0,1, \ldots, 0<\theta_{j}<1, j=1,2$ and $0<1-\theta_{1}-\theta_{2}<1$. From Theorem 2.1, we obtain a new BCR model as

$$
S_{\text {pop }}\left(y_{1}, y_{2}\right)=\frac{1-\theta_{1}-\theta_{2}}{1-\theta_{1} S_{1}\left(y_{1}\right)-\theta_{2} S_{2}\left(y_{2}\right)},
$$

with the joint cure fraction $S_{\mathrm{pop}}(\infty, \infty)=1-\theta_{1}-\theta_{2}$. The marginal survival functions are given by $S_{\text {pop }}\left(y_{1}\right)=\left(1-\theta_{1}-\theta_{2}\right) /\left(1-\theta_{2}-\theta_{1} S_{1}\left(y_{1}\right)\right)$ and $S_{\text {pop }}\left(y_{2}\right)=\left(1-\theta_{1}-\theta_{2}\right) /\left(1-\theta_{1}-\right.$ $\left.\theta_{2} S_{2}\left(y_{2}\right)\right)$, respectively. This marginal model is similar to the one proposed by $\mathrm{Gu}$ et al.[23]

In the next section, we develop a Bayesian procedure for inference on bivariate survival cure rate model given in Equation (4). Hereafter, we assume a Weibull distribution for the unobserved time $Z$ with $F_{k}\left(z \mid \boldsymbol{\gamma}_{k}\right)=1-S_{k}\left(z \mid \boldsymbol{\gamma}_{k}\right)=1-\exp \left(-z^{\gamma_{k 1}} \mathrm{e}^{\gamma_{k 2}}\right)$ and $f_{k}\left(z \mid \boldsymbol{\gamma}_{k}\right)=\gamma_{k 1} z^{\gamma_{k 1}-1} \exp \left(\gamma_{k 2}-\right.$ $z^{\gamma_{k 1}} \mathrm{e}^{\gamma_{k 2}}$ ), for $z>0, \gamma_{k 1}>0, \gamma_{k 2} \in \mathbb{R}$ and $\gamma_{k}=\left(\gamma_{k 1}, \gamma_{k 2}\right)^{\top}, k=1$, 2. However, in principle, other survival models may be undertaken, taking their specificities into account.

\section{Inference}

Let us consider the situation when the failure times $\left(Y_{1}, Y_{2}\right)$ in Section 2 are not completely observed and are subject to right censoring. Let $C_{k i}$ denote the censoring time of $k$ component, $k=1,2$. Suppose that $\left(Y_{1 i} ; Y_{2 i}\right)$ and $\left(C_{1 i} ; C_{2 i}\right)$ are independent. For each individual $i$, observed quantities are represented by the random variables $t_{k i}=\min \left\{Y_{k i}, C_{k i}\right\}$ and $\delta_{k i}=I\left(t_{k i}=Y_{k i}\right)$, which denotes a censorship indicator, $k=1,2, i=1, \ldots, n$.

Let $\boldsymbol{x}_{k i}$ denote the vectors of covariates for the $i$ th individual. Extending our model, we propose to relate the parameters $\theta_{1 i}$ and $\theta_{2 i}$ of the bivariate Poisson distribution to the covariates by the logarithmic link

$$
\theta_{1 i}=\exp \left(\boldsymbol{x}_{1 i}^{\top} \boldsymbol{\beta}_{\mathbf{1}}\right) \quad \text { and } \quad \theta_{2 i}=\exp \left(\boldsymbol{x}_{2 i}^{\top} \boldsymbol{\beta}_{\mathbf{2}}\right), \quad k=1,2,
$$

where $\boldsymbol{\beta}_{k}=\left(\beta_{k 1}, \ldots, \beta_{k p_{k}}\right)^{\top}$ is the vector of regression coefficients associated with the covariates $\boldsymbol{x}_{k i}$. Now with the expression (10) we can express the likelihood of $\boldsymbol{\vartheta}=\left(\boldsymbol{\beta}_{1}, \boldsymbol{\beta}_{2}, \boldsymbol{\gamma}_{1}, \boldsymbol{\gamma}_{2}, \theta_{0}\right)$ under non-informative censoring as,

$$
\begin{aligned}
L(\boldsymbol{\vartheta} \mid \mathcal{D})= & \prod_{i=1}^{n} S_{\text {pop }}\left(t_{1 i}, t_{2 i}\right) \times \prod_{k=1}^{2}\left[f_{k}\left(t_{k i} \mid \boldsymbol{\gamma}_{k}\right)\right]^{\delta_{k i}}\left[\theta_{0}+\left(\theta_{2 i}+\theta_{0} S_{1}\left(t_{1 i} \mid \boldsymbol{\gamma}_{1}\right)\right)\left(\theta_{1 i}+\theta_{0} S_{2}\left(t_{2 i} \mid \boldsymbol{\gamma}_{2}\right)\right)\right]^{\delta_{1 i} \delta_{2 i}} \\
& \times\left(\theta_{1 i}+\theta_{0} S_{2}\left(t_{2 i} \mid \boldsymbol{\gamma}_{2}\right)\right)^{\delta_{1 i}\left(1-\delta_{2 i}\right)}\left(\theta_{2 i}+\theta_{0} S_{1}\left(t_{1 i} \mid \boldsymbol{\gamma}_{1}\right)\right)^{\delta_{2 i}\left(1-\delta_{1 i}\right)}
\end{aligned}
$$

where $\mathcal{D}=(\boldsymbol{t}, \boldsymbol{\delta}, \boldsymbol{x}), \boldsymbol{t}=\left(\boldsymbol{t}_{1}, \boldsymbol{t}_{2}\right), \boldsymbol{t}_{k}=\left(t_{k 1}, \ldots, t_{k n}\right)^{\top}, \boldsymbol{x}=\left(\boldsymbol{x}_{1}, \boldsymbol{x}_{2}\right), \boldsymbol{x}_{k}=\left(\boldsymbol{x}_{k 1}, \ldots, \boldsymbol{x}_{k n}\right)^{\top}, \boldsymbol{\delta}=$ $\left(\boldsymbol{\delta}_{1}, \boldsymbol{\delta}_{2}\right)$ and $\boldsymbol{\delta}_{k}=\left(\delta_{k 1}, \ldots, \delta_{k n}\right)^{\top}, k=1,2$, whereas $S_{\text {pop }}\left(t_{1}, t_{2}\right)$ is survival function given in Equation (4) and $f_{k}\left(t_{k i} \mid \boldsymbol{\gamma}_{k}\right)$ and $S_{k}\left(t_{k i} \mid \boldsymbol{\gamma}_{k}\right), k=1,2$ are density and survival functions of the Weibull distribution. 


\subsection{Prior and posterior}

Now, some inferential tools are investigated under a Bayesian viewpoint. The normal distribution and gamma distribution with $a$ as shape and $b$ as scale (and mean $a / b$ ) are denoted by $N\left(\mu, \sigma^{2}\right)$ and $G(a, b)$. In this context, we assume that $\boldsymbol{\beta}_{k}, k=1,2, \boldsymbol{\gamma}_{k 1}, \gamma_{k 2}$ and $\theta_{0}$ are a priori independent, that is,

$$
\pi(\boldsymbol{\vartheta})=\prod_{k=1}^{2} \pi\left(\boldsymbol{\beta}_{k}\right) \pi\left(\gamma_{k 1}\right) \pi\left(\gamma_{k 2}\right) \pi\left(\theta_{0}\right),
$$

where $\boldsymbol{\beta}_{k} \sim N_{p_{k}}\left(\boldsymbol{\theta}, \Sigma_{k 0}\right), \gamma_{k 1} \sim \mathrm{G}\left(a_{k 0}, b_{k 0}\right), \gamma_{k 2} \sim N_{1}\left(0, \sigma_{\gamma_{k 2}}^{2}\right) k=1,2$ and $\theta_{0} \sim \mathrm{G}\left(a_{0}, b_{0}\right)$. Here, all the hyper-parameters are specified in order to express non-informative priors.

Combining the likelihood function (11) and the prior distribution in Equation (12), the joint posterior distribution for $\vartheta$ is obtained as $\pi(\boldsymbol{\vartheta} \mid \mathcal{D}) \propto L(\vartheta \mid \mathcal{D}) \prod_{k=1}^{2} \pi\left(\boldsymbol{\beta}_{k}\right) \pi\left(\gamma_{k 1}\right) \pi\left(\gamma_{k 2}\right) \phi\left(\theta_{0}\right)$. This joint posterior density is analytically intractable. Thus, the computational problem can be easily handled by using MCMC methods for sampling from the posterior distribution. From Equations (10)-(12), we write the full conditional distributions of $\boldsymbol{\beta}_{k}, \boldsymbol{\gamma}_{k}=\left(\gamma_{1 k}, \gamma_{2 k}\right)$ and $\theta_{0}$ as

$$
\begin{aligned}
& \pi\left(\boldsymbol{\beta}_{k} \mid \cdot\right) \propto \exp \left[-\sum_{i=1}^{n} \theta_{k i} F_{k}\left(t_{k i} \mid \boldsymbol{\gamma}_{k}\right)\right] \prod_{i=1}^{n} \Delta_{i} \Lambda_{k i} \pi\left(\boldsymbol{\beta}_{k}\right), \quad k=1,2, \\
& \pi\left(\boldsymbol{\gamma}_{1} \mid \cdot\right) \propto \exp \left[-\sum_{i=1}^{n}\left(\theta_{1 i} F_{1}\left(t_{1 i} \mid \boldsymbol{\gamma}_{1}\right)-\theta_{0} S_{1}\left(t_{1 i} \mid \boldsymbol{\gamma}_{1}\right) S_{2}\left(t_{2 i} \mid \boldsymbol{\gamma}_{2}\right)\right)\right] \prod_{i=1}^{n} f_{1}\left(t_{1 i} \mid \boldsymbol{\gamma}_{1}\right) \Delta_{i} \Lambda_{2 i} \pi\left(\boldsymbol{\gamma}_{1}\right), \\
& \pi\left(\boldsymbol{\gamma}_{2} \mid \cdot\right) \propto \exp \left[-\sum_{i=1}^{n}\left(\theta_{2 i} F_{2}\left(t_{2 i} \mid \boldsymbol{\gamma}_{2}\right)-\theta_{0} S_{1}\left(t_{1 i} \mid \boldsymbol{\gamma}_{1}\right) S_{2}\left(t_{2 i} \mid \boldsymbol{\gamma}_{2}\right)\right)\right] \prod_{i=1}^{n} f_{2}\left(t_{2 i} \mid \boldsymbol{\gamma}_{2}\right) \Delta_{i} \Lambda_{1 i} \pi\left(\boldsymbol{\gamma}_{2}\right),
\end{aligned}
$$

and

$$
\left.\pi\left(\theta_{0} \mid \cdot\right) \propto \exp \left[-\theta_{0} S_{1}\left(t_{1 i} \mid \boldsymbol{\gamma}_{1}\right) S_{2}\left(t_{2 i} \mid \boldsymbol{\gamma}_{2}\right)\right)\right] \prod_{i=1}^{n} \Lambda_{1 i} \Delta_{i} \Lambda_{2 i} \pi\left(\theta_{0}\right),
$$

where $\Delta_{i}=\left[\theta_{0}+\left(\theta_{2 i}+\theta_{0} S_{1}\left(t_{1 i} \mid \boldsymbol{\gamma}_{1}\right)\right)\left(\theta_{1 i}+\theta_{0} S_{2}\left(t_{2 i} \mid \boldsymbol{\gamma}_{2}\right)\right)\right]^{\delta_{1 i} \delta_{2 i}}, \Lambda_{1 i}=\left(\theta_{1 i}+\theta_{0} S_{2}\left(t_{2 i} \mid \boldsymbol{\gamma}_{2}\right)\right)^{\delta_{1 i}\left(1-\delta_{2 i}\right)}$ and $\Lambda_{2 i}=\left(\theta_{2 i}+\theta_{0} S_{1}\left(t_{1 i} \mid \boldsymbol{\gamma}_{1}\right)\right)^{\delta_{2 i}\left(1-\delta_{1 i}\right)}$.

Clearly the conditional density functions in Equations (13)-(16) do not belong to any known parametric density family. In order to generate our samples, we then implement a MetropolisHastings algorithm within Gibbs iterations.[24] For example, to implement the MetropolisHastings algorithm for the parameter $\theta_{0}$, we consider a target distribution $g_{\theta_{0}}\left(\theta_{0}\right)=\pi\left(\theta_{0} \mid \cdot\right)$, and under given model, $\theta_{0}>0$ we consider the transformation $\theta_{0}=\exp (\eta)$, where, $-\infty<\eta<\infty$. Then, $g_{\eta}(\eta)=g_{\theta_{0}}(\eta) \mathrm{e}^{\eta}$.

Instead of directly sampling $\theta_{0}$, we generate $\eta$ by choosing a normal proposal $N\left(\hat{\eta}, \sigma_{\hat{\eta}}^{2}\right)$ where $\hat{\theta}$ is the maximizer of the logarithm of $g_{\eta}(\eta)$ and $\sigma_{\hat{\eta}}^{2}$ is the minus of the inverse of the second derivative of logarithm of $g_{\eta}(\eta)$ evaluated in $\eta=\hat{\eta}$. The algorithm to generate $\eta$ operates according to (1) let $\eta$ be the current value; (2) generate a point $\eta^{*}$ from $N\left(\hat{\eta}, \sigma_{\hat{\eta}}^{2}\right)$ and (3) a move from $\eta$ to $\eta^{*}$ is made with probability $\min \left\{1,\left[g_{\eta}\left(\eta^{*}\right) \phi\left((\eta-\hat{\eta}) / \sigma_{\hat{\eta}}\right)\right] /\left[g_{\eta}(\eta) \phi\left(\left(\eta^{*}-\hat{\eta}\right) / \sigma_{\hat{\eta}}\right)\right]\right\}$, where $\phi(\cdot)$ is the standard normal probability density function.

After we sample $\eta$, we obtain $\theta_{0}=\mathrm{e}^{\eta}$. To implement the Metropolis-Hastings algorithm for the parameter $\boldsymbol{\beta}_{k}$ and $\boldsymbol{\gamma}_{k}, k=1,2$, we proceed in the same way as for the parameter $\theta_{0}$, but considering as target distribution the respective conditional density functions are given in Equations (13)-(15). 


\subsection{Model comparison criteria}

There exist a variety of methodologies to compare several competing models for a given data set and to select the one that best fits the data. One of the most used in applied Bayesian research is the deviance information criterion (DIC) proposed by Spiegelhalter et al.,[25] the expected Akaike information criterion (EAIC) - [26], and the expected Bayesian (or Schwarz) information criterion (EBIC) - [27] can be used. These criteria are based on the posterior mean of the deviance, $d(\vartheta)$ which can be approximated from the MCMC output by

$$
\bar{d}=\sum_{q=1}^{Q} \frac{d\left(\vartheta_{q}\right)}{Q}
$$

where the index $q$ indicates the $q$ th realization of a total of $Q$ realization and $d(\vartheta)=-2 \sum_{i=1}^{n} \log$ $\left[g\left(t_{1 i}, t_{2 i} \mid \vartheta\right)\right]$, where $g(\cdot)$ is the pdf corresponding to our model. For observed data, we have that $g\left(t_{1 i}, t_{2 i} \mid \boldsymbol{\vartheta}\right)$ is the $i$ th component of likelihood function.

The DIC criterion can be estimated using the MCMC output by $\widehat{\mathrm{DIC}}=\bar{d}+\widehat{\rho_{d}}=2 \bar{d}-\hat{d}$, with $\rho_{D}$ is the effective number of parameters, which is defined as $E\{d(\vartheta)\}-d\{E(\vartheta)\}$, where $d\{E(\vartheta)\}$ is the deviance evaluated at the posterior mean and estimated as

$$
\hat{D}=d\left(\frac{1}{Q} \sum_{q=1}^{Q} \boldsymbol{\beta}_{1}^{(q)}, \frac{1}{Q} \sum_{q=1}^{Q} \boldsymbol{\beta}_{2}^{(q)}, \frac{1}{Q} \sum_{q=1}^{Q} \boldsymbol{\gamma}_{1}^{(q)}, \frac{1}{Q} \sum_{q=1}^{Q} \boldsymbol{\gamma}_{2}^{(q)}, \frac{1}{Q} \sum_{q=1}^{Q} \theta_{0}^{(q)}\right)
$$

Similarly, the EAIC and EBIC criteria can be estimated by means of $\widehat{\text { EAIC }}=\bar{d}+2 \#(\vartheta)$ and $\widehat{\mathrm{EBIC}}=\bar{d}+\#(\vartheta) \log (n)$, where $\#(\vartheta)$ is the number of model parameters. The EBIC criterion used in this work is the same as that in [28]. Comparing alternative models, the preferred model is the one with the smallest criterion values.

Another criterion which is one of the most used in applied works is derived from the conditional predictive ordinate (CPO) statistic. For a detailed discussion on the CPO statistic and its applications to model selection, see Gelfand et al. [29] and Geisser and Eddy.[30] Let $\mathcal{D}$ denote the full data and $\mathcal{D}^{(-i)}$ denote the data with the $i$ th observation deleted. We denote the posterior density of $\vartheta$ given $\mathcal{D}^{(-i)}$ by $\pi\left(\vartheta \mid \mathcal{D}^{(-i)}\right), i=1, \ldots, n$. For the $i$ th observation, $\mathrm{CPO}_{i}$ can be written as

$$
\mathrm{CPO}_{i}=\int_{\vartheta \in \Theta} g\left(t_{1 i}, t_{2 i} \mid \boldsymbol{\vartheta}\right) \pi\left(\vartheta \mid \mathcal{D}^{(-i)}\right) d \vartheta=\left\{\int_{\vartheta} \frac{\pi(\vartheta \mid \mathcal{D})}{g\left(t_{1 i}, t_{2 i} \mid \boldsymbol{\vartheta}\right)} \mathrm{d} \vartheta\right\}^{-1}
$$

For the proposed model a closed form of the $\mathrm{CPO}_{i}$ is not available. However, a Monte Carlo estimate of $\mathrm{CPO}_{i}$ can be obtained by using a single MCMC sample from the posterior distribution $\pi(\vartheta \mid \mathcal{D})$. Let $\vartheta^{(1)}, \ldots, \vartheta^{(Q)}$ be a sample of size $Q$ of $\pi(\vartheta \mid \mathcal{D})$ after the burn-in. A Monte Carlo approximation of $\mathrm{CPO}_{i}[5]$ is given by

$$
\widehat{\mathrm{CPO}}_{i}=\left\{\frac{1}{Q} \sum_{q=1}^{Q} \frac{1}{g\left(t_{1 i}, t_{2 i} \mid \boldsymbol{\vartheta}^{(q)}\right)}\right\}^{-1}
$$

For model comparison, we use the log pseudo-marginal likelihood (LPML) defined by LPML $=$ $\sum_{i=1}^{n} \log \left(\widehat{\mathrm{CPO}}_{i}\right)$. The larger is the value of LPML, the better is the fit of the model. 


\section{Simulation study}

To evaluate the performance of the Bayesian estimation approach for the bivariate survival cure rate model, we carry on a simulation study. In this study, we consider the BCR model with the Weibull distribution for the event time $\left(Z_{k j}, k=1,2, j=1,2, \ldots\right)$, with parameter, $\gamma_{k 1}=1.4$ and $\gamma_{k 2}=2.0$. For each individual $i, i=1, \ldots, n$, the number of competing risks of the event of interest for this individual $\left(N_{1}, N_{2}\right)$ is generated from the bivariate distribution with parameter $\theta_{0}=0.5, \theta_{1 i}=\exp \left(\beta_{10}+\beta_{11} x_{i}\right)$ and $\theta_{2 i}=\exp \left(\beta_{20}+\beta_{21} x_{i}\right)$, where $\beta_{10}=-1.6, \beta_{11}=1.3$, $\beta_{20}=-1.5, \beta_{21}=1.1$ and the covariates $x_{i}$ are generated from a Bernoulli distribution with parameter 0.5 . The censoring times $C_{k i}$ are sampled from the uniform distribution on the interval $\left(0, \tau_{k}\right)$, where $\tau_{k}$ is set in order to control the proportion of censored observations. In this study, the proportion of censored observations was on an average approximately equal to $50 \%$.

We consider a great proportion of censored observations since it is common we find such amount of censoring in practice. For instance, a great censoring proportion is observed in the data set obtained from the diabetic retinopathy study [31] of time of blindness in each eye of diabetic patients with diabetic retinopathy, which will be considered further in Section 5.

The sample sizes are taken as $n=200$ and 400. For each simulated data set, the posterior summaries and $95 \%$ highest posterior density (HPD) intervals of the model parameters were obtained. A amount of 80,000 MCMC posterior samples are generated for each parameter, from which 20,000 iterations, regarded as the burn-in time, are eliminated for obtaining a sample of size 60,000 . The autocorrelation of these sampled values are reduced by taking a spacing of size 10 , thus resulting in 6000 samples. For each sample, we calculate the estimates of each parameter and then by using these estimates we obtain the average (AE), standard deviation (SD), the rootmean-square error (RMSE) and the coverage probability (PC). The results are all presented in Table 1, in mean values over 5000 Monte Carlo repetitions. We observe that the RMSE decreases as sample size increases. Also, we observe that the difference between the AE's and the parameter values are quite small which suggests that the estimates possess very little bias. Finally, we note that the simulated coverage probabilities are quite close to the nominal level.

\section{Application}

In this section, we fit the proposed model on a data set obtained from the diabetic retinopathy study [31] of time of blindness in each eye of the 197 diabetic patients with diabetic Retinopathy. One eye of each patient was randomly selected for treatment (the effectiveness of laser photo coagulation in delaying the onsets of blindness) and other eye was observed without treatment.

Table 1. Simulation results of the posterior summaries over 5000 replications.

\begin{tabular}{|c|c|c|c|c|c|c|c|c|c|c|}
\hline \multirow[b]{2}{*}{$n$} & & \multicolumn{9}{|c|}{ Parameter } \\
\hline & & $\beta_{10}$ & $\beta_{11}$ & $\gamma_{11}$ & $\gamma_{12}$ & $\beta_{20}$ & $\beta_{21}$ & $\gamma_{21}$ & $\gamma_{12}$ & $\theta_{0}$ \\
\hline \multirow[t]{4}{*}{200} & $\mathrm{AE}$ & -1.489 & 1.201 & 2.018 & 1.409 & -1.482 & 1.060 & 2.030 & 1.410 & 0.489 \\
\hline & SD & 0.348 & 0.381 & 0.155 & 0.161 & 0.368 & 0.345 & 0.154 & 0.153 & 0.068 \\
\hline & RMSE & 0.368 & 0.401 & 0.156 & 0.163 & 0.373 & 0.361 & 0.157 & 0.1549 & 0.072 \\
\hline & $\mathrm{PC}$ & 0.942 & 0.943 & 0.949 & 0.942 & 0.953 & 0.950 & 0.948 & 0.949 & 0.945 \\
\hline \multirow[t]{4}{*}{400} & $\mathrm{AE}$ & -1.592 & 1.283 & 2.008 & 1.391 & -1.501 & 1.010 & 2.021 & 1.403 & 0.502 \\
\hline & SD & 0.268 & 0.252 & 0.133 & 0.130 & 0.368 & 0.256 & 0.143 & 0.139 & 0.034 \\
\hline & RMSE & 0.269 & 0.254 & 0.137 & 0.131 & 0.373 & 0.258 & 0.145 & 0.141 & 0.034 \\
\hline & $\mathrm{PC}$ & 0.951 & 0.948 & 0.952 & 0.948 & 0.948 & 0.951 & 0.949 & 0.951 & 0.952 \\
\hline
\end{tabular}


A binary age covariate ( 0 for juvenile and 1 for adult) is available. The first component of the bivariate survival time is the time of the blindness on the treated eye $\left(Y_{1}\right)$ and the second component is the similar time for the untreated eye $\left(Y_{2}\right)$. One notable feature of the data set is that 159 patients experienced some form of censoring. For all these patients, censoring time is the same. For 80 patients, the censoring times were greater than or equal to the failure times of the untreated eyes. The reverse happened for only 16 patients. The remaining 38 patients experienced failure before the censoring time in both eyes of which simultaneous failures were observed for 6 patients.

Huster et al. [31] analysed the data using a frequentist approach with the Clayton-Oakes model and Weibull marginal distributions. Manatunga and Oakes [32] considered random effect models and Sahu and Dey [28] considered exponential and Weibull bivariate distributions with a Bayesian approach. and Romeo et al. [33] presented the Bayesian counterpart to the frequentist work of Shih and Louis, [34] based on Archimedean copulas with parametric estimation for the marginals. All proposals do not consider the possibility that patients who may not remain susceptible to the event of interest. Recently, Louzada et al. [16] analysed the data considering bivariate long-term distribution based on the Farlie-Gumbel-Morgenstern copula model. The objective here is to see the effect of age in the surviving of patients, and to see whether there is any association between treated and untreated time to event and to investigate whether there is any difference in the proportion of patients of non-blindness (cure fraction) between eyes treated and untreated.

Then, the BCR model proposed in Equation (4) is fitted to the data. For model fitting, the following independent priors are adopted in the Bayesian computations $\beta_{k j} \sim N\left(0,10^{4}\right), k=1,2$ and $j=0,1, \gamma_{k 1} \sim \mathrm{G}(1,0.01)$ and $\gamma_{k 2} \sim N\left(0,10^{4}\right)$. Thus, our choice is to assume weakly but informative prior. Since our prior is still informative, the posterior is always proper. A total of 80,000 MCMC posterior samples are generated for each parameter, from which the 20,000 iterations are eliminated for obtaining 60,000 samples. The autocorrelation of these sampled values are reduced by taking a spacing of size 10 thus resulting in 6000 samples. Figure 1 shows the behaviour of the chains of the estimated parameters.

Table 2 gives posterior estimates of $\boldsymbol{\beta}=\left(\boldsymbol{\beta}_{1}, \boldsymbol{\beta}_{2}\right),\left(\boldsymbol{\gamma}_{1}, \boldsymbol{\gamma}_{2}\right)$, and $\theta_{0}$, where $\boldsymbol{\beta}_{k}=\left(\beta_{k 0}, \beta_{k 1}\right)$, and $\boldsymbol{\gamma}_{k}=\left(\gamma_{1 k}, \gamma_{2 k}\right), k=1,2$. We see from Table 2 that all of the HPD intervals for the regression coefficients of the covariates contain 0 , this implies that the age do not have a significant effect on the surviving of patients. Also, from Table 2, we see that the posterior mean of $\theta_{0}$ is 0.247 , with a 95\% HPD interval of $(0.145,0.393)$ which indicates significant association between $Y_{1}$ and $Y_{2}$. A plot of the marginal posterior distribution of $\theta_{0}$ is shown in Figure 1. We see in this figure that the posterior distribution of $\theta_{0}$ appears to be slightly skewed.

To evaluate the robustness of the model with regard to the choice of the hyper-parameters of the prior distributions, a small sensitivity study is carried out with larger SDs for the prior distributions. The posterior summaries of the parameters do not display much difference and do not alter the results presented in Table 2.

We compare the fitting of the BCR model with bivariate long-term distribution based on the Farlie-Gumbel-Morgenstern (FGM) copula model [16] with Weibull mixture marginal distributions by considering DIC, EAIC, EBIC and LPLM criteria. These information criteria furnish the values given in Table 3. According to all the criteria, we have indication in favour of the BCR model.

Romeo et al. [33] modelled this data set using Archimedean copulas with Weibull marginal distribution. The estimated LPLM and BIC measures for Clayton copula were, respectively, -643.402 and 1308.02. Also, Sahu and Dey [28] considered a bivariate Weibull model, the estimated BIC measure was 1703.03. Based on Bayesian criteria, there is a clear positive evidence in favour of the BCR model, indicating that the model can be seen as a competitor to the bivariate survival with cure fraction models. 


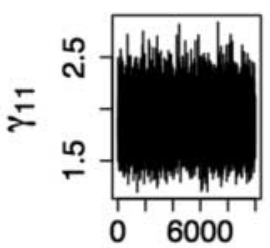

Sample

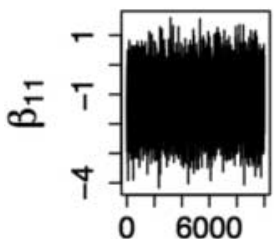

Sample

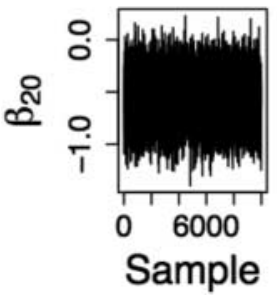

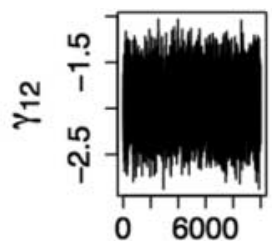

Sample

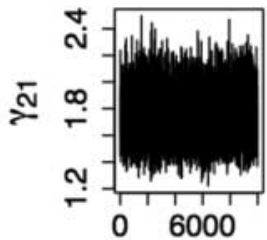

Sample

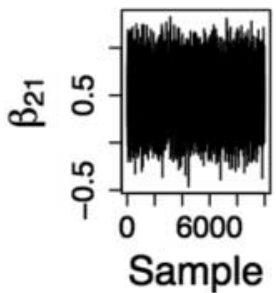

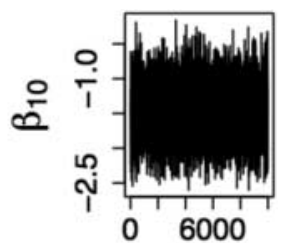

Sample

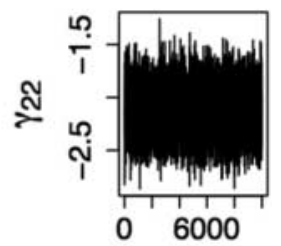

Sample

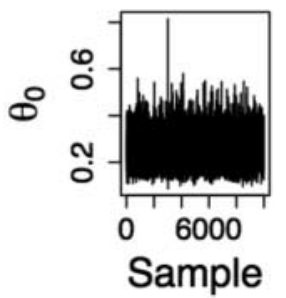

Figure 1. Sequence plots of the chains.

Table 2. Posterior summaries of the parameters for the BCR model for the diabetic retinopathy data set.

\begin{tabular}{|c|c|c|c|c|c|}
\hline \multirow[b]{2}{*}{ Time to blindness } & \multirow[b]{2}{*}{ Parameter } & \multirow[b]{2}{*}{ Mean } & \multirow[b]{2}{*}{ SD } & \multicolumn{2}{|c|}{$95 \% \mathrm{HPD}$} \\
\hline & & & & Lower & Upper \\
\hline \multirow[t]{4}{*}{ Treated eye } & $\gamma_{11}$ & 1.869 & 0.2217 & 1.473 & 2.335 \\
\hline & $\gamma_{12}$ & -1.934 & 0.2633 & -2.440 & -1.420 \\
\hline & $\beta_{10}$ & -1.449 & 0.347 & -2.129 & -0.776 \\
\hline & $\beta_{11}$ & -1.216 & 0.8207 & -2.854 & 0.380 \\
\hline \multirow[t]{5}{*}{ Untreated eye } & $\gamma_{21}$ & 1.746 & 0.165 & 1.443 & 2.101 \\
\hline & $\gamma_{22}$ & -2.112 & 0.2113 & -2.529 & -1.699 \\
\hline & $\beta_{20}$ & -0.563 & 0.221 & -0.997 & -0.129 \\
\hline & $\beta_{21}$ & 0.488 & 0.251 & -0.012 & 0.976 \\
\hline & $\theta_{0}$ & 0.247 & 0.064 & 0.145 & 0.393 \\
\hline
\end{tabular}

Table 3. Bayesian criteria for the fitted models.

\begin{tabular}{lcccc}
\hline & \multicolumn{4}{c}{ Criterion } \\
\cline { 2 - 5 } Model & LPLM & DIC & EAIC & EBIC \\
\hline FGM & -689.32 & 1589.67 & 1597.40 & 1626.95 \\
BCR & -469.12 & 932.87 & 940.92 & 970.47 \\
\hline
\end{tabular}


Table 4. Posterior summaries of the cured fraction stratified by juvenile and adult patients.

\begin{tabular}{lllcccc}
\hline & & & & \multicolumn{2}{c}{$95 \%$ HPD } \\
\cline { 5 - 6 } Eye & Patient & Mean & SD & Lower & Upper \\
\hline Treated & Juvenile & 0.611 & 0.053 & 0.498 & 0.704 \\
& Adult & 0.710 & 0.063 & 0.565 & 0.805 \\
\multirow{2}{*}{ Untreated } & Juvenile & 0.430 & 0.059 & 0.320 & 0.551 \\
& Adult & 0.309 & 0.060 & 0.195 & 0.428 \\
\hline
\end{tabular}

We turn our attention to the role of the covariates on the cured fraction $p_{0}$. Table 4 shows the posterior summaries of the cured fraction stratified by group (juvenile and adult). A plot of the marginal posterior distribution of $\theta_{0}$ is shown in Figure 2. The plot and the table both reveals that the mean cure fraction for old patients with treated eye is higher than that of juvenile patients. In

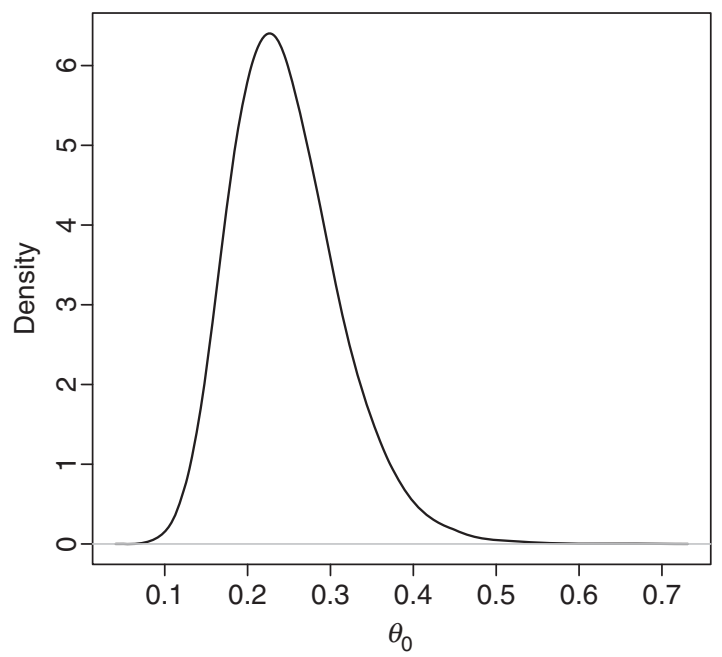

Figure 2. The marginal posterior density of $\theta_{0}$.
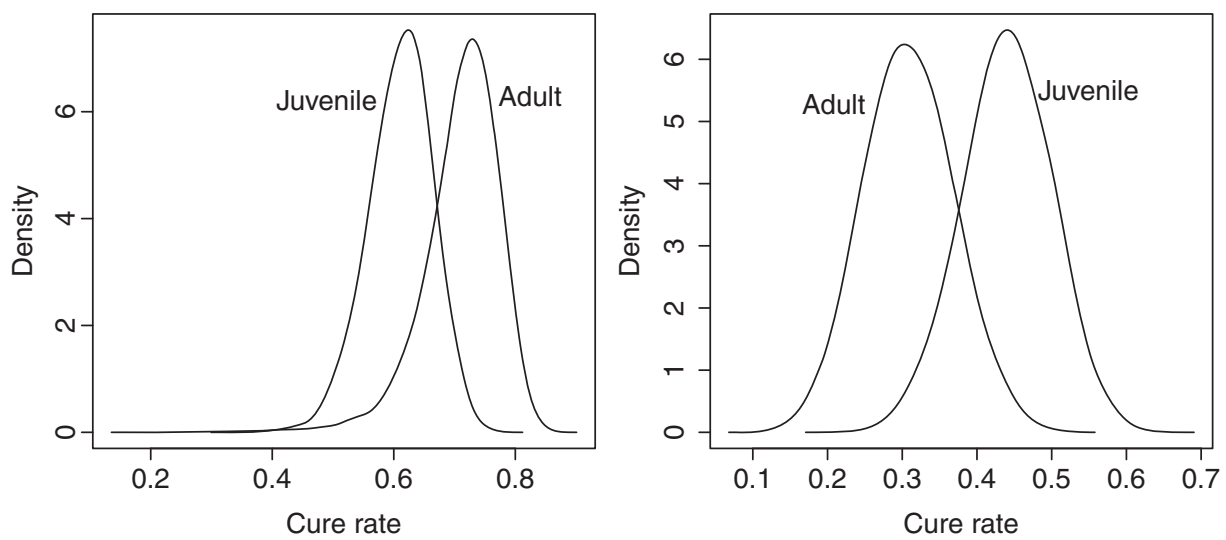

Figure 3. Posterior density estimates for the cure rates corresponding to treated eye (left panel) and untreated eye (right panel) patients. 

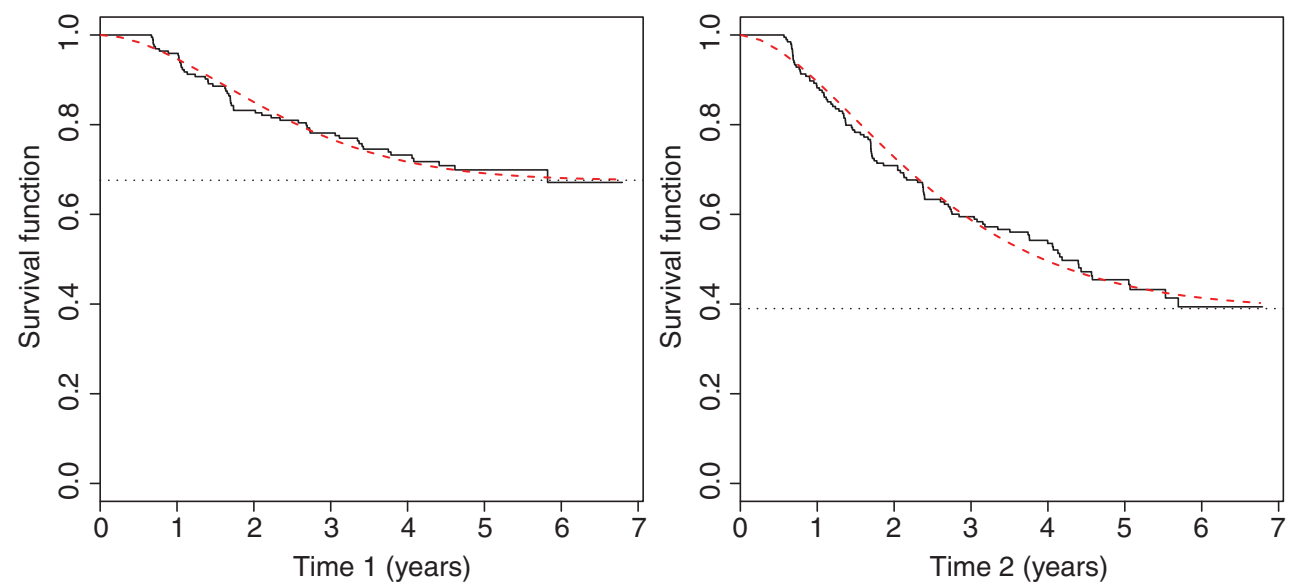

Figure 4. Kaplan-Meier curve together with Bayesian estimates of the survival for patients with treated eye (left panel) and untreated eye (right panel).

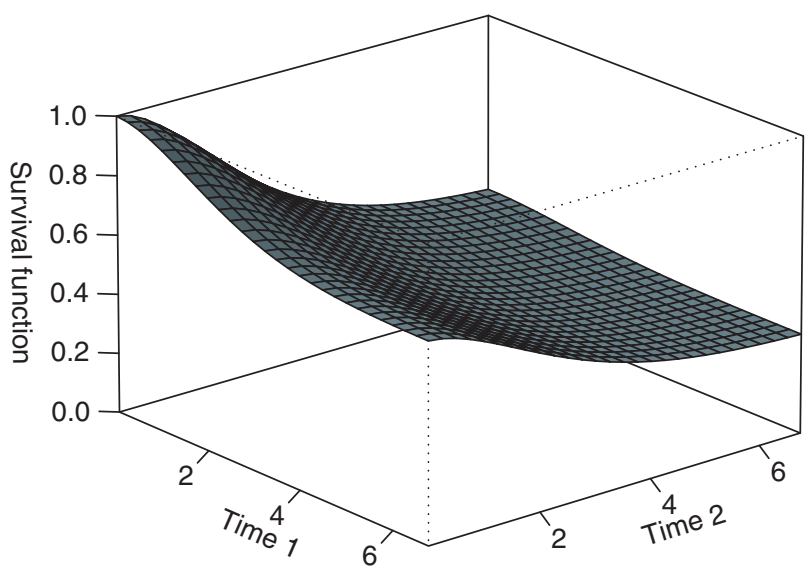

Figure 5. The bivariate posterior survival surface.

the case of juvenile patients with untreated eye the mean cure fraction is higher than that of the old patients. For both age groups, in the case of patients with treated eye the mean cure fraction is greater than that of the patients with untreated eye (Figure 3).

Figure 4 exhibits the Kaplan-Meier estimates of the survival function together with the Bayesian estimates of the marginal survival function based on the multivariate cure rate model. The covariates are not used in constructing plots. We see that the two curves in both plots on the left and right panel are nearly similar and appear to plateau after approximately 6 years of follow-up. Figure 5 shows a three-dimensional plot of the posterior mean survival surface. We observe in this plot how the survival curve plateaus for each failure time variable.

\section{Conclusions}

In this paper, we proposed a new multivariate survival model with cure rate and have examined some of its properties. This model is useful for jointly modelling any type of time to event data with a surviving fraction. We also develop a multivariate extension of the univariate survival 
cure rate model proposed by Tsodikov et al. [7] and Rodrigues et al.[22] In the application to the diabetic retinopathy data set, we discovered that the BCR model provides the best fit.

The model considered in this article can be fitted using standard available software,[35] which makes the approach quite powerful and accessible to practitioners in the field.

Future developments of our work may involve other parametric or semi-parametric extensions of the multivariate lifetime distribution under the proposed set-up, as well as, the relaxation of assumption that $Y_{k}=\min \left\{Z_{k 0}, Z_{k 1}, \ldots Z_{k N_{k}}\right\}$. Indeed, we may consider the modelling of its counterpart, $Y_{k}=\max \left\{Z_{k 0}, Z_{k 1}, \ldots Z_{k N_{k}}\right\}$, corresponding to a complementary risk scenarios as discussed by Louzada-Neto.[36] Moreover, following Cooner et al.,[37] we envisage a generalization of our framework by assuming $Y_{k}$ as random, such as we may scan all possible $Y_{k}$ values from the first to last order statistics.

\section{Disclosure statement}

No potential conflict of interest was reported by the authors.

\section{References}

[1] Berkson J, Gage RP. Survival curve for cancer patients following treatment. J Am Statist Assoc. 1952;47:501-515.

[2] Boag JW. Maximum likelihood estimates of the proportion of patients cured by cancer therapy. J R Statist Soc B. 1949;11:15-53.

[3] Yakovlev AY, Tsodikov AD. Stochastic models of tumor latency and their biostatistical applications. Singapore: World Scientific; 1996.

[4] Maller RA, Zhou X. Survival analysis with long-term survivors. New York: Wiley; 1996.

[5] Ibrahim JG, Chen M-H, Sinha D. Bayesian survival analysis. New York: Springer; 2001.

[6] Chen M-H, Ibrahim JG, Sinha D. A new Bayesian model for survival data with a surviving fraction. J Am Statist Assoc. 1999;94:909-919.

[7] Tsodikov AD, Ibrahim JG, Yakovlev AY. Estimating cure rates from survival data: an alternative to two-component mixture models. J Am Statist Assoc. 2003;98:1063-1078.

[8] Tournoud M, Ecochard R. Application of the promotion time cure model with time-changing exposure to the study of HIV/AIDS and other infectious diseases. Stat Med. 2007;26:1008-1021.

[9] Cancho VG, Rodrigues J, de Castro M. A flexible model for survival data with a cure rate: a Bayesian approach. J Appl Stat. 2011;38(1):57-70.

[10] Perdona GC, Louzada-Neto F. A general hazard model for lifetime data in the presence of cure rate. J Appl Stat. 2011;38:1395-1405.

[11] Kim S, Chen M-H, Dey DK. A new threshold regression model for survival data with a cure fraction. Lifetime Data Anal. 2011;17(1):101-122.

[12] Cobre J, Perdoná GSC, Peria FM, Louzada F. A mechanistic breast cancer survival modelling through the axillary lymph node chain. Stat Med. 2013;32:1536-1546.

[13] Chatterjee N, Shih J. A bivariate cure-mixture approach for modeling familial association in diseases. Biometrics. 2001;57(3):779-786.

[14] Price DL, Manatunga AK. Modelling survival data with a cured fraction using frailty models. Stat Med. 2001;20(910):1515-1527.

[15] Chen M-H, Ibrahim JG, Sinha D. Bayesian inference for multivariate survival data with a cure fraction. J Multivariate Anal. 2002;80(1):101-126.

[16] Louzada F, Suzuki AK, Cancho VG. The FGM long-term bivariate survival copula model: modeling, Bayesian estimation, and case influence diagnostics. Commun Stat - Theory Methods. 2013;42(4):673-691.

[17] Karlis D. An em algorithm for multivariate poisson distribution and related models. J Appl Stat. 2003;30(1):63-77.

[18] Caroni C, Crowder M, Kimber A. Proportional hazards models with discrete frailty. Lifetime Data Anal. 2010;16(3):374-384.

[19] Cox D, Oakes D. Analysis of survival data. London: Chapman and Hall; 1984.

[20] Clayton DG. A model for association in bivariate life tables and its application in epidemiological studies of familial tendency in chronic disease incidence. Biometrika. 1978;65(1):141-151.

[21] Oakes D. A model for association in bivariate survival data. J R Statist Soc Ser B (Methodol). 1982;414-422.

[22] Rodrigues J, Cancho VG, de Castro M, Louzada-Neto F. On the unification of long-term survival models. Stat Probab Lett. 2009;79:753-759.

[23] Gu Y, Sinha D, Banerjee S. Analysis of cure rate survival data under proportional odds model. Lifetime Data Anal. 2011;17(1):123-134.

[24] Chib S, Greenberg E. Hierarchical analysis of sur models with extensions to correlated serial errors and timevarying parameter models. J Econ. 1995;68(2):339-360. 
[25] Spiegelhalter DJ, Best NG, Carlin BP, van der Linde A. Bayesian measures of model complexity and fit. J R Statist Soc: Ser B (Statist Methodol). 2002;64:583-639.

[26] Brooks SP. Discussion on the paper by Spiegelhalter, Best, Carlin, and van der Linde (2002). J R Statist Soc B. 2002;64:616-618.

[27] Carlin BP, Louis TA. Bayes and empirical Bayes methods for data analysis. 2nd ed. Boca Raton (FL): Chapman and Hall/CRC; 2001.

[28] Sahu SK, Dey DK. A comparison of frailty and other models for bivariate survival data. Lifetime Data Anal. 2000;6(3):207-228.

[29] Gelfand A, Dey D, Chang H. Model determination using predictive distributions with implementation via sampling based methods (with discussion). In J. Bernardo JM, Berger JO, Dawid AP, Smith AFM, editors. Bayesian statistics, Vol. 4. Oxford: Oxford University Press; 1992. p. 147-167.

[30] Geisser S, Eddy W. A predictive approach to model selection. J Am Statist Assoc. 1979;153-160.

[31] Huster W, Brookmeyer R, Self S. Modelling paired survival data with covariates. Biometrics. 1989;145-156.

[32] Manatunga AK, Oakes D. Parametric analysis for matched pair survival data. Lifetime Data Anal. 1999;5(4):371387.

[33] Romeo JS, Tanaka NI, Pedroso-de-Lima AC. Bivariate survival modeling: a Bayesian approach based on copulas. Lifetime Data Anal. 2006;12(2):205-222.

[34] Shih J, Louis T. Inferences on the association parameter in copula models for bivariate survival data. Biometrics. 1995;1384-1399.

[35] R Core Team. R: a language and environment for statistical computing. Vienna: R Foundation for Statistical Computing; 2012.

[36] Louzada-Neto F. Poly-hazard regression models for lifetime data. Biometrics. 1999;55:1121-1125.

[37] Cooner F, Banerjee S, Carlin BP, Sinha D. Flexible cure rate modeling under latent activation schemes. J Am Statist Assoc. 2007;102:560-572. 